cardiac CT that are summarised in Abstract 111 table 1. These incidental findings resulted in further investigations, documented in Abstract 111 table 2. The mean radiation dose ( \pm SEM) for CAC scoring was $0.61 \pm 0.03 \mathrm{mSv}$. The mean radiation dose ( \pm SEM) for subsequent CTCA was $2.66 \pm 0.32 \mathrm{mSv}$ in high pitch "flash" mode $(\mathrm{n}=27), \quad 5.86 \pm 0.50 \mathrm{mSv}$ in prospective mode $(\mathrm{n}=64)$ and $17.15 \pm 1.68 \mathrm{mSv}$ in the retrospective mode $(\mathrm{n}=25)$.

Abstract 111 Table 1 Incidental findings on cardiac CT

\begin{tabular}{lllr}
\hline Area & Structure & Incidental Finding & $\mathbf{n}$ \\
\hline Chest $(\mathrm{n}=27)$ & Lung parenchyma & Nodule $<1 \mathrm{~cm}$ & 5 \\
& & Emphysema & 3 \\
& & Atelectasis & 6 \\
& & Fibrosis & 4 \\
& & Tumour recurrence & 1 \\
& Pleura & Bronchiectasis & 2 \\
& & Effusion & 2 \\
Abdomen $(\mathrm{n}=7)$ & Calcification & 2 \\
Diaphragm $(\mathrm{n}=5)$ & Lymph node & Adenopathy & 2 \\
Vasculature $(\mathrm{n}=11)$ & Adernal & Cyst/Nodules & 6 \\
& & Adenoma/metastasis & 1 \\
& Aorta & Hiatus Hernia & 5 \\
& & Dilatation & 8 \\
& Renal & Aneurysm & 1 \\
& Coeliac & Stenosis & 1 \\
& & Stenosis & 1 \\
\hline
\end{tabular}

Abstract 111 Table 2 Further investigation of incidental findings on Cardiac CT

\begin{tabular}{lr}
\hline Investigation & $\mathbf{n}$ \\
\hline Bone scintigraphy & 1 \\
Chest clinic referral & 2 \\
CT chest & 4 \\
DMSA & 1 \\
MR adrenals & 1 \\
MRA renal & 1 \\
Nephrology clinic referral & 1 \\
Pleural fluid aspiration & 1 \\
Ultrasound kidneys & 1 \\
Ultrasound liver & 3 \\
\hline
\end{tabular}

Abstract 111 Table 3 Investigations and referrals generated by incidental findings

\begin{tabular}{lc}
\hline Investigations or referrals & Number \\
\hline Bone scintigraphy & 1 \\
Chest clinic referral & 2 \\
CT chest & 4 \\
DMSA & 1 \\
MR adrenals & 1 \\
MR cardiac & 2 \\
MRA renal & 1 \\
Nephrology clinic referral & 1 \\
Pleural fluid aspiration & 1 \\
Ultrasound kidneys & 1 \\
Ultrasound liver & 3
\end{tabular}

Conclusions Despite 62 patients having a reassuring CAC score of zero, $8 \%$ of this group had evidence of non-calcified plaque, with one patient having obstructive $\mathrm{CAD}$ that required intervention. We conclude that if strong clinical suspicion remains in patients with a CAC score of zero further coronary investigation may be warranted. Incidental findings are common, and can result in multiple further investigations for patients. Further research is needed to evaluate the added cost, clinical benefits and radiation exposure created by investigation of such incidental findings in the context of cardiac CT.

\section{COMPUTED TOMOGRAPHIC CORONARY ANGIOGRAPHY TO SCREEN FOR ALLOGRAFT VASCULOPATHY AFTER HEART TRANSPLANTATION}

doi:10.1136/heartjnl-2011-300198.112

M G Panicker, A G Mitchell, N R Banner, T K Mittal. Harefield Hospital, Harefield, UK

Objective To evaluate ComputedTomographic Coronary Angiography (CTA) as an alternative to Invasive Coronary Angiography (ICA) for the detection of Cardiac Allograft Vasculopathy (CAV).

Background CAV is an important cause of late mortality after heart transplantation (HT). Because patients are often asymptomatic, surveillance ICA is performed in our institution. CTA is effective for the diagnosis of coronary disease in non-transplant patients, but few studies have been done after HT.

Methods $117 \mathrm{HT}$ patients, 1 to 24 years post transplant (mean=12 years SD \pm 6 ) underwent CT coronary artery calcification (CTCAC) followed by retrospective ECG gated coronary angiogram on a 64slice scanner without the use of any $\beta$-blockers. Majority (89\%) of patients had CTA within $24 \mathrm{~h}$ before ICA. The Agatston calcium score (CS) was calculated for all patients. The CTA images were systematically analysed for image quality and the presence of CAV (graded as significant if $>50 \%$ luminal stenosis) using a fifteen coronary segments model by an independent investigator blinded to the results of ICA.

Results CS ranged from 0 to 1681 (Mean=91.7 \pm 275 ). Out of 77 patients with absent CS, 3 had significant CAV on ICA. Despite a mean resting heart rate of $82 \mathrm{bpm} S D \pm 13$ and body mass index of $27 \mathrm{~kg} / \mathrm{m}^{2} \mathrm{SD} \pm 5,81 \%$ of the CTA images were graded as excellent or satisfactory. For all the 1755 segments assessed by CTA irrespective of the image quality, CTA had sensitivity, specificity, positive and negative predictive values of $71 \%, 79 \%, 72 \%$ and $78 \%$ respectively for the detection of any CAV found by ICA. On a patient basis, CTA best performed in diagnosing CAV of more than $25 \%$ with sensitivity, specificity, positive and negative predictive values of $74 \%, 94 \%, 79 \%$, and $92 \%$ respectively. None of the 61 patients with completely normal CTA had CAV on ICA. $83(92 \%)$ out of 90 patients who responded to a patient survey preferred CTA to ICA as a screening test for CAV. Non-coronary cardiac and non-cardiac abnormalities were identified in $18 \%$ and $14 \%$ patients respectively.

Conclusion The study shows that CTA compares favourably with ICA in detecting CAV in heart transplant recipients, and may be a preferable screening technique because of its non-invasive nature, patient preference and yield of additional information. One has to exercise caution in just using CS in these patients as significant CAV can be missed out.

\section{DUAL ENERGY CT IMPROVES DIFFERENTIATION OF CORONARY ATHEROSCLEROTIC PLAQUE COMPONENTS COMPARED TO CONVENTIONAL SINGLE ENERGY CT}

doi:10.1136/heartjnl-2011-300198.113

${ }^{1} \mathrm{D}$ R Obaid, ${ }^{1} \mathrm{P}$ A Calvert, ${ }^{1} \mathrm{~J} \mathrm{H}$ F Rudd, ${ }^{2} \mathrm{D}$ Gopalan, ${ }^{1} \mathrm{M}$ R Bennett. ${ }^{1}$ University of Cambridge, Cambridge, UK; ${ }^{2}$ Papworth Hospital NHS Trust, Cambridge, UK

Introduction Vulnerable plaques have a relatively high necrotic core area and low fibrous tissue content. Although CT can identify plaque components on the basis of their x-ray attenuation, there is 
significant overlap between their attenuation ranges, most crucially between necrotic core and fibrous plaque. Recently introduced dual energy CT (DECT) permits acquisition of 2 different energy data sets simultaneously, with the change in attenuation of plaque components to different energies depending upon their material composition. We therefore examined whether DECT was better than single energy CT in determining plaque components defined by virtual histology IVUS.

Methods 20 patients underwent DECT and 3-vessel VH-IVUS. CT data was obtained at peak voltages of $100 \mathrm{kV}$ and $140 \mathrm{kV}$. 52 plaques were chosen with either homogenous fibrous plaque or confluent areas of calcified plaque or necrotic core as defined by VH-IVUS. VHIVUS images were co-registered and orientated with the corresponding CT images using distance from coronary ostia and fiduciary markers (Abstract 113 figure 1). Multiple regions of interest (ROI) were placed within the plaque components or in lumen on cross sectional CT images pre-classified by VH-IVUS (Abstract 113 figure 1). ROI densities were measured (in Hounsfield Units) and assigned to the plaque component. A dual energy index (DEI) was created for each component, defined as the ratio of the difference in attenuation at 2 different energies / sum of attenuation with 1000 added to each attenuation value to avoid negatives.

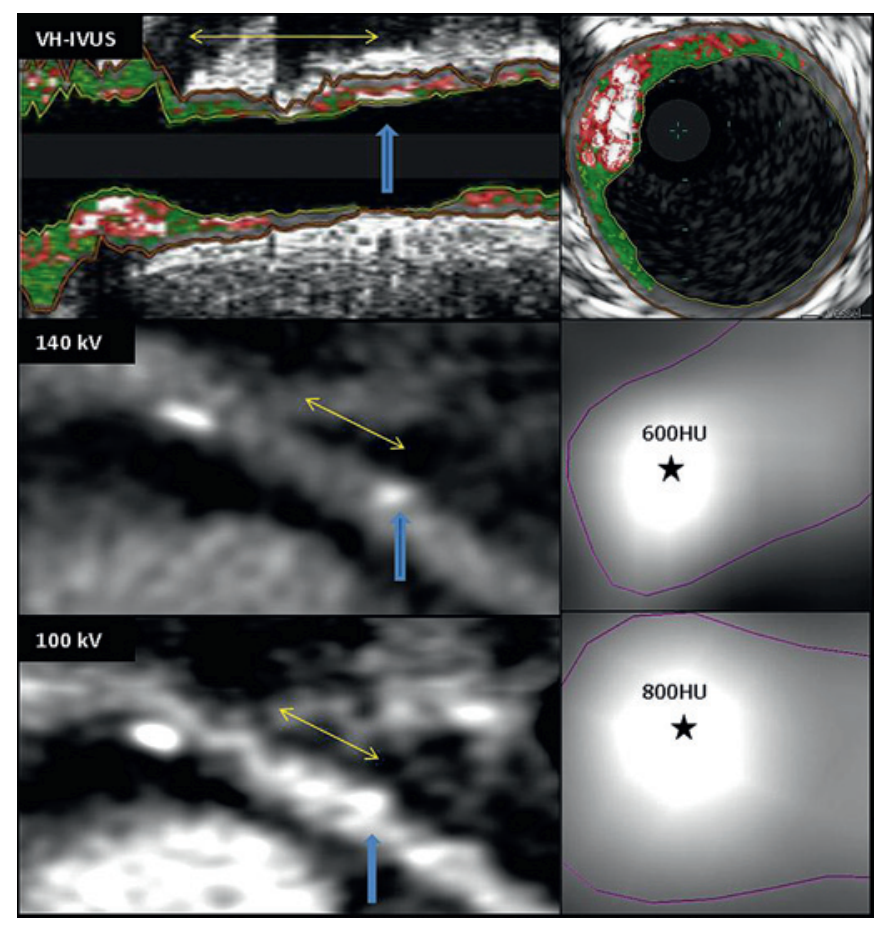

Abstract 113 Figure 1 Demonstration of plaque co-registration between VH-IUS and $140 \mathrm{kV} / 100 \mathrm{kV}$ CT data sets. Calcified plaque is identified $5 \mathrm{~mm}$ from side branch adjacent to characteristic calcification (yellow line). Cross section taken through this plaque (blue arrow) and following orientation with VH-IVUS cross section HU region of interest sampling is performed in calcified plaque.

Results Attenuation values for 1088 ROIs were measured from 70 paired data sets at $100 \mathrm{kV}$ and $140 \mathrm{kV}$ creating 70 DEIs (12 necrotic core, 11 fibrous plaque, 29 calcified plaques and 18 lumen). Values obtained using a single energy data set showed good differentiation between calcified plaque and all others $(p<0.05)$, but considerable overlap between necrotic core and fibrous plaque $(p=n s)$ (Abstract 113 figure 2A) (Abstract 113 table 1). In DECT, lumen (iodinated contrast) showed the greatest change in attenuation and hence had the highest DEI. Necrotic core had the lowest DEI and could be distinguished from all other components $(p<000.1)$ Importantly, in contrast to the single energy data, DEI derived from both energy data sets permitted resolution of necrotic core and fibrous plaque without overlap (Abstract 113 figure 2B).
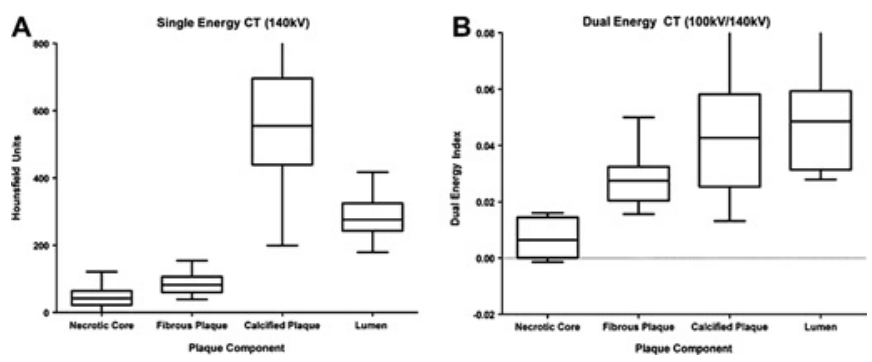

Abstract 113 Figure 2 (A) Defined CT attenuation spectra of plaque components using a single energy $(140 \mathrm{kV})$, calcified plaque is distinguishable from all others but necrotic core and fibrous plaque overlap. (B) The use of dual energy index from the attenuation data at 2 energies $(100 / 140 \mathrm{kV})$ allows significant separation of necrotic core and fibrous plaque $(p<0.05)$ (Tukeys multiple comparison test).

Abstract 113 Table 1

\begin{tabular}{lcccl}
\hline $\begin{array}{l}\text { Plaque } \\
\text { Component }\end{array}$ & $\begin{array}{l}\text { 100 kV mean } \\
\text { HU (SD) }\end{array}$ & $\begin{array}{l}\text { 140 kV mean } \\
\text { HU (SD) }\end{array}$ & $\begin{array}{l}\text { Mean Difference } \\
(\mathbf{1 0 0}-\mathbf{1 4 0} \mathbf{~ k V})\end{array}$ & $\begin{array}{l}\text { Dual Energy } \\
\text { Index (mean) }\end{array}$ \\
\hline Necrotic Core & $57.26(42.20)$ & $42.69(31.51)$ & 14.57 & 0.0071 \\
Fibrous Plaque & $148.30(49.47)$ & $84.60(30.34)$ & 63.69 & 0.0283 \\
Calcified Plaque & $733.10(226.7)$ & $582.20(194.9)$ & 150.9 & 0.0450 \\
Lumen & $411.5(82.27)$ & $282.90(55.93)$ & 128.6 & 0.0483 \\
\hline
\end{tabular}

Conclusions The additional attenuation data provided by DECT improves the differentiation of plaque components when compared to conventional single energy CT. In particular, DECT may allow better differentiation of necrotic core and fibrous plaque, a weakness of conventional cardiac CT, allowing for more accurate non-invasive identification of vulnerable plaques.

\section{RADIATION DOSES TRENDS FROM CARDIAC CT USING A CARDIAC SPECIFIC CONVERSION FACTOR: SYSTEM UNDERSTANDING \& AN OPTIMISATION STRATEGY SIGNIFICANTLY REDUCES THE DOSE TO THE PATIENTS IN A CLINICAL SERVICE}

doi:10.1136/heartjnl-2011-300198.114

${ }^{1} \mathrm{O}$ E Gosling, ${ }^{1} \mathrm{~S}$ Iyengar, ${ }^{1} \mathrm{R}$ Loader, ${ }^{1} \mathrm{G}$ Morgan-Hughes, ${ }^{2} \mathrm{~W} D$ Strain, ${ }^{3} \mathrm{C}$ Roobottom. ${ }^{1}$ Plymouth Hospitals NHS Trust, Plymouth, UK; ${ }^{2}$ Peninsula College of Medicine and Dentistry, Exeter, UK; ${ }^{3}$ Peninsula College of Medicine and Dentistry, Plymouth, UK

Background CT coronary angiography CTCA now has an established role in the investigation of patients with chest pain. Under the IRMER regulations radiation doses to patients should be kept as low as reasonably practical (ALARP). Previous publications have used a chest conversion factor to calculate the effective dose ( $\mathrm{mSv}$ ) from CTCA. We have previously demonstrated that chest conversion factors significantly under-estimate the effective dose to the patient when applied to CTCA and have calculated a cardiac specific conversion factor of $0.028 \mathrm{mSv}$ (mGy.cm)-1. Our department follows the ALARP ethos and has implemented new technologies together with physician training to reduce the radiation dose from CTCA. We aimed to investigate what impact the implementation of new technologies has had on the radiation dose of CTCA.

Method All patients who were coded as attending for a cardiac CT scan on the PACS and CRIS systems were included in the analysis Scan indication included: rule out coronary artery disease, CABG assessment, pre-EP studies and problem solving. CT scanning 